quires notice here is the composition of the hepatic tissue in another form of disease, which, however, did not occasion dropsy. The patient after repeated attacks of syphilis was seized with dysentery, followed first by stricture of the rectum, and subsequently by ulceration of the colon and artificial anus. He died with rapid emaciation and diarrhœea. His liver was much enlarged, of a uniform pale yellowish-brown colour, and of the consistence of new cheese. When subjected to analysis, Dr Bostock found that it consisted entirely of a fatty matter resembling tallow, deposited in meshes of cellular tissue. The contents of the gall.bladder were a puriform mucus, quite destitute of bilious intermixture. Dr Bostock, we may add, has also given the analysis of the fluid of the gall-bladder in a case of protracted jaundice, accompanying tubercular liver and dropsy. It had an orange-red colour, and thin consistence; the animal matter in it was almost entirely albumen, with a little colouring matter different from that of the bile; and in fact it contained none of the usual biliary principles.

We must defer the consideration of the rest of Dr Bright's work till next number.

ArT. II.-Transactions of the Association of Fellows and Licentiates of the King and Queen's College of Physicians in Ireland. Vol. V. Dublin, 1828. pp. 576.

T

HE members of the Irish Association continue to promote with energy the purposes of their institution. If in the present volume the communications are more varied and numerous than in those formerly published, they are in no degree less valuable, either in improving the accuracy, or in extending the boundaries of medical science. It contains twenty articles on various subjects in medical and surgical pathology and therapeutics.

The first papers to which the attention of our readers may be directed, are the "Clinical Observations of Dr Robert Reid on the Epidemic Fever of 1826," and the "Report of the House of Recovery and Fever-Hospital of Dublin, for the year ending in 182\%, by Dr John O'Brien." In the former the fever is described as it was observed in the Fever and Dysentery-Hospital, Kevin Street ; in the latter, as it occurred in the House of Recovery and Fever-Hospital of Cork Street. The information communicated by both may be condensed in the following manner.

Fever, which is never absent in some degree from large cities, prevails almost every season in Dublin to a very great 
extent, and assumes occasionally a form so violent as to resemble rather a sweeping pestilence than the usual manageable form of ordinary fever. The year 1826 afforded one of those seasons during which this disease spread with such rapidity and so extensively among the population of Dublin, as never to have been equalled in its effects by any previous epidemic hitherto recorded. Whether the meteorological peculiarities of the season had any real influence in communicating this epidemic character is perhaps not a point susceptible of strict demonstration. Dr O'Brien, however, thinks it not unimportant to record, that the spring of 1826 was cold and dry, the summer intensely hot and dry, the autumn variable, and the winter severe and inclement; - a succession of weather which all testimony, historical and traditional, concur in representing as insalubrious to Ireland. He further remarks, that the injury which the potato crop sustained during the unusually hot dry summer and autumn of 1825, with the want of employment and consequent poverty of the working population, subjected a large proportion to the miseries of famine. The knowledge of these circumstances led a committee, appointed to inquire into the state of the poor, to apprehend an aggravation of public calamity in the probable addition of epidemic disease; and the result showed that their fears were not without foundation.

According to Dr Reid, epidemic cases of the disease began to appear so early as the end of February or the beginning of March 1826. In April the number of applications at the Cork Street Fever-Hospital had considerably increased. On the 9th of May the number of applications exceeded the means of accommodation, which amounted to 220 beds; and this increase augmented so rapidly as early in June to attract the serious attention of the public authorities. On the 17th the Board of Health addressed to the physicians of the different hospitals of Dublin circulars, requesting information as to the causes, character, and extent of the fever, and on the adequacy of the means of relief within the reach of those among whom chiefly its prevalence was observed. One of the principal points in the answers of the physicians was the inadequacy of hospital accommodation, and the consequent diffusion of the disease by the sick remaining too long among the healthy. To obviate this evil, such measures were adopted by government as the urgency of the occasion demanded, and the promptitude with which the means of alleviation required to be administered.

In the garden of the Meath Hospital a shed or temporary building was erected, and was opened on the 4th August for the reception of 240 patients. On the 15 th of the same month the accommodation of the Cork Street Hospital was, by the 
erection of tents in the lawn, capable of containing 180 patients, augmented from 220 to 400 . On the 18th the Wellesley Hospital, North King Street, was opened for the reception of 113 patients. Further accommodation was provided at Sir Patrick Dun's and at Steven's Hospitals; and when all these measures proved insufficient for the exigencies of the season, a large building in Kevin Street was fitted up and opened on the 4th September for the reception of 230 patients. By these measures the hospital accommodation was enlarged at this time to

\begin{tabular}{lll} 
Cork Street Hospital, & & 400 \\
Meath Hospital Garden, & - & 240 \\
Wellesley Hospital, & - & 113 \\
Kevin Street Hospital, & - & - \\
& & $\mathbf{2 3 0}$ \\
\hline
\end{tabular}

By these several means the total amount of hospital accommodation was augmented to above 1400 beds, or 400 more than that, which was found requisite during the epidemic of 181\%-18. If to this it be added, that there still remained so many of the sick poor at their own houses, that, in a public meeting held at the Mansion House, on the 26th October, to consider the best means of arresting the progress of the epidemic, it was stated, upon accurate documents by the Lord Mayor, that 3200 persons were under the influence of the disease at their own houses, it will convey an idea of the extent of epidemic sickliness greatly more aggravated than any thing to which we are accustomed on this side of St George's Channel.

During this month (October) the fever, which had been gradually extending its influence, was thought to have attained its highest rate. This it preserved during the months of November and December; but in January 1827 the first marks of abatement occurred in the reappearance of ague of various forms ; and in the beginning of March it underwent a sudden and unexpected decline, which continued uninterrupted through the months of April and May. About the conclusion of the former, the abatement was so considerable as to admit the evacuation and final removal of the sick-camp on the 8th of May. On the 12th, the number in hospital was only 185 , scarcely exceeding the usual average. At the end of May or the beginning of June, it is considered by Dr Reid as having entirely ceased.

The commencement of the epidemic presented two forms of fever, distinguished by Dr O'Brien into the old and new constitution.

The first was the ordinary typhus of this country, proceeding to the 11th, 14th, 17th, or 21st days. Though inferior 
in numerical amount to the one immediately to be noticed, it was much more fatal. In several instances, however, it was complicated with the latter, and assumed its symptoms. The delirium was then more violent, amounting to frenzy. Black crusts on the tongue, teeth, and gums were seldom seen. Petechiæ were less frequent; but the skin in many cases became yellow, though not of the deep tint observed in icteroid or yellow fever. The temperature of the surface was little increased, and was often even lower than the natural standard; and the general aspect of the fever partook more of the nervous than of the vascular character.

The second variety, or that of the new constitution, which was most predominant, was one distinguished chiefly by short periods, terminating in $3,5,7$, or 9 days, the five-day period being the most common. Though in hospital practice it is difficult, in consequence of the confusion of recollection in most cases of fever, to distinguish accurately the day of invasion, in the present epidemic the crisis was generally ascertained with facility. In the months of June and July 1826, Dr O'Brien, after cautious observation of the critical day in 40 cases, found 19 terminated on the 5 th day, 12 on the $7 \mathrm{th}, 5$ on the 11 th, 2 on the 17 th, and 2 on the 21 st days. The invasion, which usually came on at noon, consisted of a sudden attack of sickness, headach, pain in the lumbar region, and chillness. Toward evening, when all the symptoms increased, the chillness became a rigor, and the sickness was followed by vomiting or retching, continuing more or less for 3 or 4 days, sometimes through its entire course. On the evening of the 5 th or 7 th days, generally after a rigor, sometimes without previous warning, profuse sweating continuing during the night completed the crisis, and left the patient convalescent in the morning. In this comparatively mild form of the disease, the most perplexing circumstance was the frequency of relapses, which were so common as to give rise to the idea, that in several instances the fever was one of a long period broken into short ones. The liability to relapse is stated to be in the inverse ratio of the duration of the disease; the five-day fevers being more prone to relapse than the seven-day, the seven than the nine, and so forth. The apyrectic period is stated to be longer in the seven-day than in the five-day fevers, yet to be irregular in all, varying from 24 hours to a fortnight or upwards. To prove that the relapsing tendency was not under the influence of irregularities in diet, Dr O'Brien adduces the fact, that nearly all the nurse-tenders, who were "6 treated with the utmost attention and kindness," suffered one or more relapses. Is it not possible, that, if operating in the form of indulgences, kindness may have occasionally proved an exciting 
cause of relapse? The sulphate of quina, the author, from its effects in some cases, is inclined to regard as a beneficial preventive.

It is a feature of some interest in the history of this epidemic, that its character and phenomena appear to have been much modified by the season. Thus during summer it was distinguished by the intense affection of the vascular system, more especially of that of the brain; hard, rapid pulse; acuteheadach, and active delirium; and pains in the muscles and bones, sometimes with a light yellow tint of the skin. The disease was nevertheless generally mild, since whole families, labouring under all the disadvantages of filth and poverty, are said to have passed through it with impunity. In a few only the symptoms characteristic of typhus appeared. But in general, while the diffusion of this variety was great, its mortality was small.

Somewhat different was the character of the disease during autumn and winter, when dysenteric symptoms, either concomitant or successive, tended to complicate the disease, and add considerably to its fatality. Of this complication the worst forms were accompanied by intestinal hemorrhage degenerating sometimes into melæna; and from this few aged persons recovered. As the winter advanced a change took place in the type of the fever, in the 5th and 7th day fever being converted into periods of 11 and 14 days, or even more protracted. The tendency to relapse diminished at the same rate.

Of the accidental concomitants of the fever the most important was gangrene of the feet and toes, which took place in four instances among the patients in the tents, in consequence of the intensity of the cold during the months of December and January. Of these three proved fatal from the gangrene alone; and in one, after the feet sloughed off, recovery took place under the use of wine and cordials, and after removal into a comfortable ward.

As the winter proceeded the character of the disease again underwent some change. Already it was beginning to betray less tendency to spread, and to be limited chiefly to the persons originally attacked. But towards spring it showed a distinct tendency to intermit and to remit; and ague even, which had for years been a stranger in Dublin, began to prevail generally. The manner in which this transition took place appears to have been chiefly in the cases of continued fever undergoing relapses, which invariably observed the intermittent form sometimes irregularly, sometimes the regular quotidian or tertian.

The following table exhibits at a single view the rate of increase and decrease of the fever, and its mortality at different periods of its progress :- 
Transacions of the Irish Association.

1826. Ad Admitted.|Discharged.|Died.

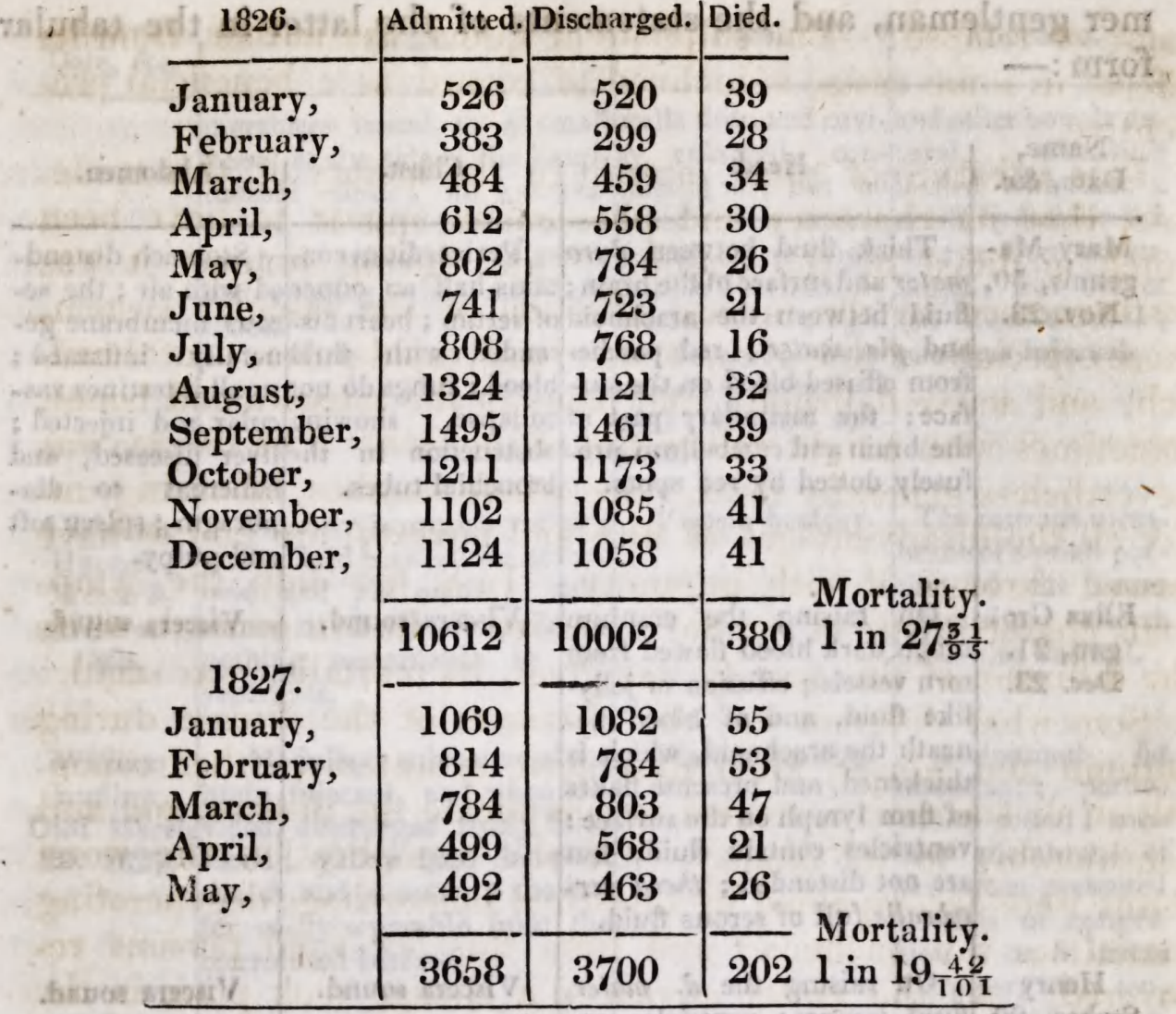

Total mortality, 1 in $23 \frac{51}{5} \frac{6}{2}$

The following table shows the comparative influence of the hospital and the tents over the mortality of the epidemic :-

From 4th Aug. to 4th Sept. 5th Sept. to 4th Oct. 5 th Oct. to 4 th Nov. 5th Nov. to 4th Dec. 5th Dec. to 4th Jan. 1827. 5 th Jan. to 4 th Feb. 5th Feb. to 4th March, 5th Mar. to 4th April,

\begin{tabular}{|c|c|c||c|c|c|}
\multicolumn{2}{|c|}{ House. } & \multicolumn{3}{|c|}{ Tents. } \\
\hline Adm. & Dis. & Died. & Adm. & Dis. & Died. \\
\hline 660 & $\frac{610}{16}$ & $\frac{16}{432}$ & 262 & 7 \\
925 & 922 & 28 & 570 & 539 & 11 \\
753 & 730 & 18 & 458 & 443 & 15 \\
702 & 692 & 22 & 403 & 392 & 19 \\
663 & 627 & 28 & 461 & 424 & 13 \\
655 & 662 & 37 & 414 & 420 & 18 \\
511 & 487 & 29 & 303 & 297 & 24 \\
538 & 534 & 26 & 246 & 269 & 21 \\
\hline 5407 & 5264 & 204 & 3286 & 3046 & 128
\end{tabular}

The information derived from dissection in this epidemic was limited by the regulations of the Cork Street Fever Hospital, which do not permit examinations. To supply this defect, however, Dr O'Brien gives the results of six inspections at the hospital of Sir Patrick Dun, and Dr Reid gives those of seven in the Kevin Street Hospital. We give the table of the for- 
mer gentleman, and the statements of the latter in the tabular form

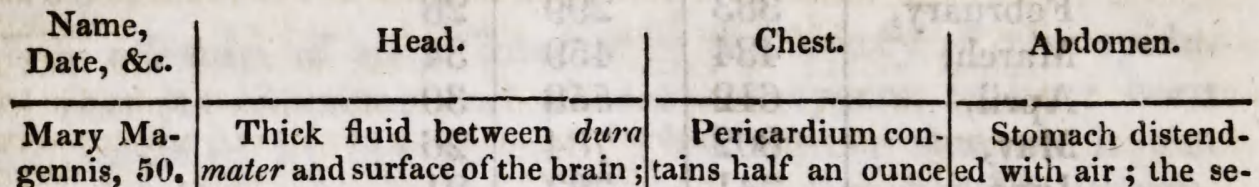

Nov. 23. fluid between the arachnoid of serum; heart dis-rous membrane geand pia mater; red patches tended with fluid nerally inflamed; from effused blood on the sur- blood; lungs do not small intestines vasface; the medullary part of collapse, showing cular and injected; the brain and cerebellum pro-obstruction in the liver diseased, and fusely dotted by red spots. bronchial tubes. adherent to diaphragm ; spleen soft and pulpy.

Eliza Gro- On raising the cranium gan, 21. much dark blood flowed from Dec. 23. torn vessels ; effusion of jelly. like fluid, and of blood beneath the arachnoid, which is thickened, and presents flakes of firm lymph on the surface; ventricles contain fluid, but are not distended ; theca vertebralis full of serous fluid.

Henry On raising the d. mater, Stokes, 20. Guid escapes; ventricles conFeb. 12, tain one ounce of fluid ; ves1827. sels injected; substance of brain very firm.

B. Gahan, Thick fluid beneath $d$. $w_{0} a$ 43. March ter; fluid between arachnoid

17, 1826. and $p$. mater; brain firmer than natural. Fluid in ventricles, and at the base of the brain.

William

Black, 23.

October 3, 1826.

Mary

Arachnoid opaque ; jellyDevine, 20. like fluid beneath, most abunDec. 27, dant at the top of the hemis. 1826.

Ann Watpheres; vessels not very turgid; but brain firm.

son, 19.

D. mater extremely vascuronchial tubes.

\section{Viscera sound. Viscera sound.}

Viscera sound.

Viscera sound.

Viscera sound.

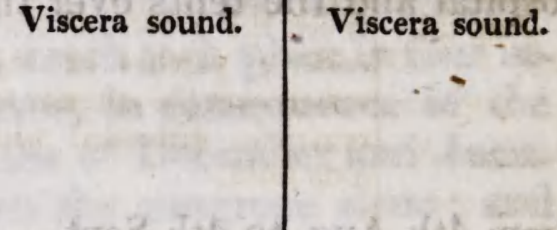

Viscera sound; Gastric mucous bronchial mucous membrane blood. membrane unusual-shot, and marked ly vascular. $\quad$ by patches of effused blood; cuodenum and jejunum similar ; liver, \&ce. bealthy.

Viscera sound. Viscera sound.

Jan. 9, coagulable lymph covering the fat much red fluid 1827. Died entire right hemisphere, and in the pericardium; membrane of small on 13th. depressing its middle; he-lleft ventricle flabby; arch; duodenum, 


\begin{tabular}{|c|c|c|c|}
\hline $\begin{array}{c}\text { Name, } \\
\text { Date, \&c. }\end{array}$ & Jit azsul Head. & & n. \\
\hline 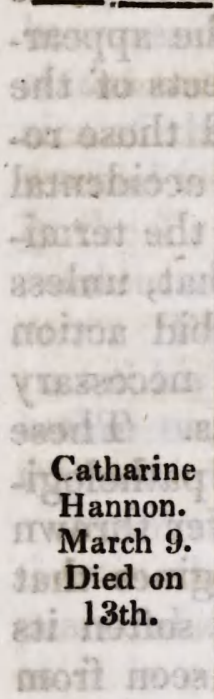 & $\begin{array}{l}\text { morrhage traced to a small } \\
\text { vessel at the side of the longi- } \\
\text { tudinal sinus ; no extrava- } \\
\text { sation in left hemisphere ; } \\
\text { cerebral substance injected, } \\
\text { and effusing, when cut, fluid } \\
\text { blood. } \\
\text { D. mater brownish yellow; } \\
\text { yellow fluid between the arach- } \\
\text { noid and pia mater; sub- } \\
\text { stance of the brain yellow; } \\
\text { nothing remarkable in the } \\
\text { ventricles. }\end{array}$ & $\begin{array}{l}\text { walls thin, and cavi- } \\
\text { ty enlarged, con- } \\
\text { laining air, but no } \\
\text { blood; left auricle } \\
\text { containing air; right } \\
\text { auricle distended by } \\
\text { venous blood; right } \\
\text { v. natural ; inner } \\
\text { surface of aorta dyed } \\
\text { pink; texture soft- } \\
\text { ened. } \\
\text { Viscera healthy. }\end{array}$ & $\begin{array}{l}\text { and other bowels na- } \\
\text { tural ; omentum } \\
\text { loaded with fat; a } \\
\text { small hydatid in left } \\
\text { ovary ; liver not en- } \\
\text { larged, but deeper } \\
\text { red than natural ; } \\
\text { pancreas injected. } \\
\text { The mucous mem- } \\
\text { brane of a small por- } \\
\text { tion of the ileum } \\
\text { injected ; viscera } \\
\text { otherwise natural. }\end{array}$ \\
\hline $\begin{array}{c}\text { William } \\
\text { Ludlow. } \\
\text { Died March } \\
\text { 29, } 1827 . \\
\end{array}$ & $\begin{array}{l}\text { Medullary substance of the } \\
\text { brain injected, and when di- } \\
\text { vided, discharged freely fluid } \\
\text { blood; yellow fluid between } \\
\text { the } d \text {. and } p \text {. mater ; the lat- } \\
\text { ter easily separable from the } \\
\text { convoluted surface. }\end{array}$ & Viscera healthy. & $\begin{array}{l}\text { Abdominal fat } \\
\text { abundant; perito- } \\
\text { næum sound; mu- } \\
\text { cous membrane of } \\
\text { the ileum presented } \\
\text { patches of conges- } \\
\text { tion } 4 \text { or } 5 \text { inches } \\
\text { diameter, with ten- } \\
\text { dency to ulceration. }\end{array}$ \\
\hline $\begin{array}{l}\text { Owen Bra- } \\
\text { dy, hotel- } \\
\text { waiter. Died } \\
\text { Jan. 23. }\end{array}$ & $\begin{array}{l}\text { Serous blood discharged } \\
\text { when separating the scull } \\
\text { cap; glairy fluid found be- } \\
\text { tween the arachnoid and pia } \\
\text { mater; patches of injected } \\
\text { vessels over the anterior lobes; } \\
\text { ventricles distended with se- } \\
\text { rous fluid. }\end{array}$ & Viscera healthy. & $\begin{array}{l}\text { Liver such as is } \\
\text { seen in persons ha- } \\
\text { bituated to excess in } \\
\text { spirituous liquors. }\end{array}$ \\
\hline $\begin{array}{l}\text { Peter Wil- } \\
\text { liams. }\end{array}$ & 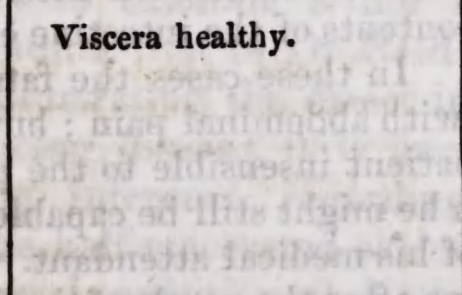 & $\begin{array}{l}\text { A large quantity } \\
\text { of purulent matter } \\
\text { like curds of cream } \\
\text { in left pleura; no } \\
\text { adhesions; right } \\
\text { lung disorganized; } \\
\text { old adhesions. }\end{array}$ & $\begin{array}{l}\text { Viscera healthy. } \\
\text {. }\end{array}$ \\
\hline Master- & Not examined. & Lungs healthy. & $\begin{array}{r}\text { Abdomen tumid; } \\
\text { integuments green ; }\end{array}$ \\
\hline 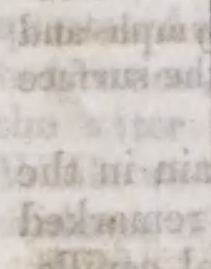 & $1 x=$ & yibit & $\begin{array}{l}\text { inferior parts of } \\
\text { trunk and extremi- } \\
\text { ties quite black; pe- } \\
\text { ritonæal cont of the } \\
\text { viscera covered with } \\
\text { a yellow curd like } \\
\text { matter; much pus }\end{array}$ \\
\hline
\end{tabular}

and sanies in the cavity; liver studded with a crystalline miliary eruption, similar to that which appeared on the back and shoulders during the disease. 
Though we here place before the reader these results of necroscopic examination, we cannot deny that they throw little new light on the intimate nature of the disease. The appearances detailed by Dr O'Brien indicate rather the effects of the febrile process than the nature of that process ; and those recorded by Dr Reid are to be viewed principally as accidental consequences supervening during the course or after the termination of that process. It may indeed be argued, that, unless the fever had preceded, these appearances of morbid action would not have followed. The febrile action was a necessary condition to the production of the subsequent effects. These effects are regarded, in the spirit of some of the old pathological notions revived by Dr Reid, as the result of fever thrown upon some peculiar texture or organ. Thus he imagines that the febrile action falling on the arterial system may soften its texture and dispose to hemorrhage, as is sometimes seen from the vessels of the nose and rectum.

"When the result of the disease," Dr Reid proceeds to say, "has fallen on the substance of the brain, this organ after death exhibited a most remarkable state of congestion. On cutting the medullary mass a quantity of thin blood, apparently in a dissolved state, was poured from innumerable points. These cases were by far the most rapidly mortal. Some have occurred to my observation when this state of the brain was about to commence, and before many minutes elapsed the progressive changes towards death took place, while I was standing by the patient. In all the cases of this kind which I have examined there were observed patches of several inches in extent of a dark colour upon the ileum. Upon opening this intestine these patches were found to be occasioned by a state of vascular congestion in the mucous surface, sometimes even apparently abrasions were observed. Indeed, in one case there were several perforations, so that the contents of the intestine escaped into the cavity of the peritonæum. In these cases the fatal train of symptoms generally commenced with abdominal pain; but the state of the brain soon rendered the patient insensible to the progress of disease in the abdomen, although he might still be capable of giving a rational reply to the questions of his medical attendant. When the patients have survived a day or two after the result of the disease has been deposited upon some internal part, bands of adhesion were observed; but in the rapidly fatal cases the coagulable lymph and curd-like substance could be wiped off the organ, while the surface - appeared sound."

The escape of fluid blood from sections of the brain in the foregoing account demonstrates, as was long ago well remarked by Morgagni, the highly injected state of the cerebral capillaries. The occurrence of inflammation and ulceration of the intestinal mucous membrane in the same cases is an interesting fact, confirming the observations of Petit and Serres on the entero-mesenteric fever, those of Andral, and those of Breton- 
neau. A similar confirmation we have occasion to notice in the pathological researches of Dr Bright ; and perhaps it would be unreasonable now to question the principle, though originally stated too unconditionally by Broussais, that one of the tendencies of the febrile process is to produce inflammation of the intestinal villous membrane. That this inflammation affects chiefly the Peyerian glands is a point which may be regarded as established by the researches of Petit, and Serres, and Bretonneau. To this subject, however, Dr Reid appears not to have directed his attention. As far as can be discovered from the account before us, this morbid state of the intestinal villous membrane appears to have taken place towards the close of the disease.

From the little communicated regarding the peritonæal affection in the conclusion of the preceding extract, this also it appears takes place towards the termination of the disease. But as it is certain that this peritonæal inflammation is not a uniform consequence of fever, it would be very desirable to ascertain the circumstances under which it most generally takes place. To say, as Dr Reid seems to do, that it is a deposit or result of the febrile action, obviously does not explain its origin. For were it an essential or genuine result, it would take place in all cases uniformly. Upon the whole, it may be doubted whether it can be viewed in any other light than as peritonæal inflammation succeeding to and complicating the previous febrile attack.

The opinions of both authors on the etiology, or the causes concerned in the production of fever, it is scarcely possible to notice with propriety. For though these opinions, like many others, are illustrated with much ingenuity, they are from their nature not susceptible of very strict demonstration; and it is unnecessary to examine either their merits or their defects. While we admit that to Dr Reid we are indebted for one valuable fact, illustrating the direct influence of putrid animal matter in inducing disease, it is impossible to derive from it any very strong inference regarding the production of fevers: Though the half-macerated skeletons of the well of the Palace of the Holy Inquisition at Valladolid were in all probability the direct cause of the putrid fever with which the regiment quartered in that building was harassed, it is almost unnecessary to mention, that putrid fever appears in many situations in which the water is free from all contamination of this description. While we admit the value of the fact, we desire more perfect evidence of its general and uniform operation.

It is just, nevertheless, to remark, that the theoretical views now stated have suggested to Dr Reid the internal exhibition of a remedial agent, the active powers of which promise a valuable improvement and simplification in the treatment of several dan- 
gerous diseases. Reflecting on the principle which Dr Reid conceives correct, that all the deleterious and disorganizing effects of fever result from the operation of a septic morbid poison on the animal textures, he was led to infer that an agent capable of preventing the formation of this poison within the system, or of neutralizing its effects, was likely to be the most efficacious antidote to the disease. The antiseptic powers of the chloride of lime at once suggested that substance as the most likely to fulfil the object required; and of the remedial effects of this agent he first satisfied himself in dysentery. Having thus ascertained the sanative powers of chloride of lime in bad cases of dysentery, four of which are recorded at the conclusion of the paper, Dr Reid attempted the same remedy in cases of fever which usually proceed rapidly to a fatal termination, and with the most beneficial result. The conditions which Dr Reid appears to think indicated the exhibition of this remedy were while the patient had low muttering delirium, dry, yellow, or brown furred tongue, subsultus, small creeping pulse, with a faint purple dirty colour of the cheeks. The manner of using the medicine, which Dr Reid after some trial prefers, is to dissolve 3 ounces of dry chloride of lime in a pint of water, stirring occasionally for 24 hours, and filtering. The strength of this solution, as ascertained by a solution of indigo in sulphuric acid, varied from the rate of 9 to 12 drops, which were requisite to discharge the colour of 25 drops of the indigo solution.

The administration of the hydro-chloride, however, is suited to a particular stage only of fever. In all other respects the treatment by both physicians appears to have been conducted on the usual principles now recognized as the most efficacious. The several branches of the antiphlogistic and cooling regimen in the form of blood-letting, general and local, purgatives, antimonial medicines, shaving the head and the application of cold, are the measures which appear to have been most generally adopted. When depletion had been carried to a great length, Dr O'Brien found the restorative influence of wine in moderate doses highly beneficial in removing the lingering symptoms of fever, and re-establishing the capillary circulation.

The contagious character of this fever was demonstrated in the most unequivocal manner. Of $5^{17}$ nurse-tenders employed at Cork Street Hospital for the $\mathbf{1 2}$ months previous to May 1827, 52 were attacked by fever; and of this number 15 had second attacks, that is, not relapses, but fevers with long intervals interposed. It is nevertheless a proof of the small mortality of this epidemic, that of the individuals now mentioned 2 only died. The registrar and matron suffered attacks of fever within the period now defined, and the apothecary underwent 
a severe assault of febrile dysentery. Of 60 permanent residents in the institution 55 passed through the epidemic disorder.

Among the sufferers by this epidemic, we regret to find the name of Dr Stack, physician to Sir Patrick Dun's hospital, who fell a victim to a second attack of the disorder, caught during the exercise of his professional duties.

Connected with this subject may be mentioned the observations of Dr Jacob on "Internal Inflammation of the Eye following Typhus Fever."

Of this disease, which was originally noticed by $\mathrm{Mr}$ Hewson in his work on venereal ophthalmia, Dr Jacob met with between 70 and 80 examples during the epidemic, which forms the subject of the papers already noticed. It consists not merely in iritic inflammation, but in a general affection of the whole internal part of the eye, and especially of the retina. Though it appeared in some instances before the patient left the hospital, in the majority of cases it took place within 6 weeks or 2 months after recovery; more rarely it appeared 4,5 , or even so late as 8 months after. It was more frequent in the young than in the old. Of 30 cases three only were above 25 ; and in no case was it seen at a later period than 45 years.

Confined in all the cases hitherto remarked to one eye, its commencement was betrayed by musce volitantes, clouds, and luminous coils or stars,--symptoms which denote disturbance in the circulation of the retina. These in the course of 6,8 , or 10 days, sometimes longer, were followed by the sensation of a black cloud or film before the eyes, when vision seems to have become gradually impaired. About the same time, or soon after, the aqueous humour became turbid, the iris contracted and inflamed; and with much pain and intolerance of light, the usual marks of iritic inflammation, the pink vascular zone became fully manifest. In the worst forms of the disease the lens became partially opaque, reflecting oblique light, and presenting an opaline amber colour; and if this proceeded, vision was generally destroyed. Though purulent matter was occasionally secreted in the anterior chamber of the aqueous humour, constituting hypopyon, Dr Jacob never observed either tubercular masses of lymph or abscesses, which are believed to distinguish the syphilitic form of inflammation.

Besides the usual antiphlogistic means calculated to subdue local inflammation and organic irritation, Dr Jacob uses extract of belladonna very freely with the constitutional influence of mercury. Two grains of calomel, with $\frac{1}{4}$ th grain of opium three times daily, or 3,4 , or 5 grains of the blue pill three times daily for 8 or 10 days, generally produced, with mercurial 
action, decided relief. Of sulphate of quinine, though used by others with advantage, he speaks doubtfully.

The second article to which we shall request attention is a "6 Pathological Inquiry into the nature of Hydrocephalus, grounded on an attentive observation of the phenomena and of the appearances presented on dissection," by Thomas Mills, M. D. \&c.

This paper constitutes one of the most instructive collections of facts illustrating the pathology of hydrocephalus, acute and chronic, that has hitherto appeared. Excellent as are the sketches of Rush and Quin, the works of Cheyne, Yeats, Golis, Carmichael Smith, and Dr Monro on this subject, it is impossible to refuse the present essay the merit of being the best practical commentary on all that has yet been written on this disease. When originally communicated to the association the essay consisted of 20 fatal cases of acute hydrocephalus, and 14 fatal cases of the chronic disease with the necroscopic appearances, and 8 cases of well-marked hydrocephalus, terminating favourably under suitable treatment. From each class of the cases, fatal and favourable, 8 were withdrawn at the request of the committee of publication, so that at present the paper consists of 12 cases of the acute, and 6 of the chronic form, with necroscopic reports, and short but judicious pathological commentaries, and $\mathbf{2}$ only of hydrocephalus terminating favourably.

On the details of these cases, reports, and comments, it is foreign to our purpose to dwell at length. It is sufficient to mention the principal results to which they lead.

Hydrocephalus Dr Mills distinguishes, like most authors, into acute and chronic, the former varying in duration from 7 to 28 days, the latter from 1 to 6 months. He admits, however, that in the former it may run its course in 3 or 5 days, while in the latter it may be protracted to $1,2,3$, or even 16 years.

Of the 20 acute cases 12 died before they obtained the age of 6 ; seven between the 6 th and 8 th year; and one at the age of 12. Of the 14 cases of the chronic form, 7 died before the age of 4 ; three between 7 and 8 ; and four between 8 and 16 years. Of 34 patients, therefore, nearly two-thirds died before completing the 6 th year. The average of these cases shows the acute form to be more prevalent than the chronic in the ratio of 10 to $\%$; and of recoveries 7 out of 10 laboured under the acute form of the disease.

It appears also that these cases establish with considerable certainty a fact which has been hitherto stated with vague generality,-that hydrocephalic effusion is connected with the strumous diathesis. They show that though the disease may attack the healthy children of healthy parents, it is greatly 
more frequent in the puny and scrofulous, or in children whose parents are scrofulous, debilitated, or worn out by intemperance and irregularity. Traces of strumous disease were found in 22 of the patients examined. These traces were found in the brain in 2 ; in the lungs in 3 ; in the liver in 4 ; in the mesenteric glands in 8 ; in the spleen in 4 ; and in the cervical glands in 5. Of those who recovered 6 had no visible marks of scrofula ; and the parents of 26 were free from any appearance of this description.

Though Dr Mills concludes from the phenomena of cases, and from the appearances presented on dissection, that in hydrocephalus the brain is the organ primarily engaged, he nowhere gives any very precise view of the nature of the affection. It is, however, manifest from the general tenor of his comments and reflections, that he regards the acute form at least as an inflammatory disease. To the opinion that it is an inflammatory disorder of the brain there is this objection, - that inflammation is known to produce in that substance either pulpy destruction (ramollissement,) or suppuration either in cells or in a cyst. Dr Mills seems not to be aware, so far as we can perceive, that the necroscopic reports which he gives in the cases before us, tend very strongly to confirm an opinion which has been familiar to us for some time, and which, we perceive, was also advanced some time ago on the continent,-that the pathological cause of hydrocephalus, acute or chronic, is congestion or inflammation affecting the capillaries of the pia mater, and especially that division of it, which, under the name of choroid plexus, enters the ventricles of the organ. It is deserving of particular notice, that of the 12 fatal cases in 11 the pia mater was more or less vascular, sometimes highly so, and in all with effusion between it and the arachnoid membrane. In one (10) it presented coagulable lymph ; in one (3) it presented slight adhesion at the falx ; and in one (4) the dura mater, that is, its arachnoid covering is stated to be vascular. In one only (5) was the pia mater natural. In four of the dissections the cho roid plexus is said to be highly vascular or turgid with blood; in two it was pale, in one of which the pia mater, and in the other the walls of the ventricles, were highly vascular. In the rest the state of this part of the cerebral membrane is not remarked, an omission to be regretted, considering the injected state of the pia mater, connected with the serous effusion into the cavities. In the same manner we find in the six chronic cases that the pia mater was in all highly vascular, sometimes with effusion of coagulable lymph, and that in two the choroid plexus was very vascular. It is further worthy of notice, that in a small proportion only was the substance of the brain much vol. $\mathrm{xxx}$. No. 96 . 
injected, and in none were any of the usual consequences of inflammation of that organ recognized. Upon the whole, though it must not be concealed that the positive evidence in favour of this inference is not perfectly complete, it is still sufficiently strong to justify the conclusion, that injection of the capillaries of the cerebral membranes is the most uniform anatomical character of the disease, which terminates in effusion into the ventricles of the brain.

On the treatment of this malady Dr Mills delivers very judicious instructions. Blood-letting, general and local, either by opening the temporal artery, the jugular vein, or the brachial, or by cupping or by leeches, cathartics, antimonials, and calomel with opium, in the order now observed, are the therapeutic measures on which chiefly he relies for the extinction of the unhealthy action. Of opium, he prefers the "watery extract" to the ordinary tincture, as less heating, less narcotic, and more directly sedative. In the treatment of the chronic form he recommends milder measures upon the whole, more liberal exhibition of mercury, more sparing employment of opium, and the use of the means of counter-irritation.

The paper is concluded with observations on the method of prophylaxis, in which he is desirous to strike at the root of the evil, by correcting the constitution and habits of the parents. Like many other observers, he finds the causes of all our maladies in the luxurious, intemperate, irregular, or unnatural habits of civil life ; and with the view of counteracting their pernicious effects, he recommends foreign residence or travel. Unlike the majority of counsellors on this topic, however, he sends not his patient to the rich fields of France, the vineyards and citron groves of Spain, the classical shores of Italy, or the islands of the Mediterranean. There he conceives vice and luxury, misery and discontent, despotism and slavery equally concur to defeat the object of search. To enjoy at once the advantages of the most perfect, virtuous, and happy form of society, and the beauties of the natural world in unsophisticated purity, he energetically recommends the United States of America. The merits of this advice it is unnecessary to examine seriously. However favourable the varied climate and society of the Union may be to the increase of population, it is certain that diseases of scrofulous origin are neither unknown nor unfrequent.

The next paper to which we shall direct attention, entitled "Observations on the Peripneumonia of Children, by Dr Thomas Cumming," forms a valuable history of a disease of considerable interest to the practitioner. * On this malady the first information of much importance was communicated by Dr Cheyne in 1809, and $\operatorname{Dr}$ Hastings in 1820; and it is chiefly with the 
view of adding something to the facts established by these writers that Dr Cumming has digested the result of his experience in the present communication.

Though affecting children of all ages from a few days old to 8 or 9 years, it occurs most frequently between 9 months and 2 years. In children above this Dr Cumming observed it to be generally a sequel of measles, or a complication of hooping cough. Approaching much like catarrh, it presents, when fully formed, marks of a severe lesion of the lungs. The respiration is hurried, laborious, wheezing, and so rapid as to range between 60 and 100 in the minute, with frequent short dry cough, flushing of the face succeeded by a livid swollen appearance, and hard and sometimes full pulse, varying in frequency from 168 to 200. As the disease proceeds, the breathing becomes still more laborious and panting; the wheezing is converted into mucous rattling, with inability to cough; to the livid look of the countenance are added drowsiness and inability to move; the pulse becomes intermitting and imperceptible; the extremities cold; and in the course of 8 or 10 days from the commencement of the attack death takes place by suffocation. When the respiration descends from 70,80 , or 100 to 54 , or 48 in the minute, it generally indicates a favourable termination. This takes place generally about the 5th, 6th, or 7th day ; and the disease is then rarely protracted beyond a week. When it is continued longer without terminating fatally, it passes into the chronic form, with puriform expectoration, cough, wasting, and the usual signs of pulmonary consumption.

Inspection demonstrates the pathological character of this disorder. The most frequent morbid appearance is increased solidity of the lung, varying from mere vascular injection to complete hepatization. Of this change the posterior inferior portion is the most frequent seat; and while the upper portion may be healthy, the lower may be completely hepatized. The affected lung is then purple-red externally, bright-red and granular in substance, void of crepitation, firm, solid, and sinking in water. The third degree of Laennec, or that of purulent infiltration, Dr Cumming has not met with in this disease.

With the change now mentioned are combined in every case almost traces of bronchial inflammation. The tracheobronchial membrane is red, injected, and covered with viscid sero-mucous fluid ; -in cases more advanced with puriform mucus, preventing collapse of the lung. In two cases in which no traces of inflammation could be detected in the trachea or bronchial tubes, the air-cells were filled with puriform mucus, which exuded on making sections of the lung. In one case only of hooping-cough, combined with inflammation of the pulmonic 
tissue terminating fatally by convulsions, was the mucous membrane of the windpipe, bronchial tubes and cells, free from apparent disease. The more intense the marks of inflammation of the bronchial membrane, and the more considerable the effusion, the less in general is the induration, and conversely.

In a few instances the pleura presented albuminous and seroalbuminous effusions, adhesion, and other marks of inflammation.

In two instances, the lungs were thickly studded with small gray tubercles; and in another, the exterior of each lung presented many tubercles, several of which were in a state of suppuration. In both the pulmonic tissue was indurated, and the bronchial membrane inflamed. In the two former subjects, tubercles were found on the pleura and peritonæum; and the bronchial and mesenteric glands were enlarged; and in one the spleen was as thickly occupied with tubercles as the lungs.

In three instances in which the head was examined serum was found in the ventricles and in the base of the brain.

From the necroscopic appearances above detailed, Dr Cumming justly infers that the disease is a catarrhal or bronchial peripneumony; - a combination of bronchial and pneumonic inflammation. He further infers, from the incipient symptoms being generally catarrhal, that it consists at first in inflammation of the bronchial membrane, which is speedily propagated to the submucous or pulmonic tissue.

For the proper treatment of this disorder, Dr Cumming lays down three indications; 1 st, to arrest the inflammation before it has proceeded to effusion; $2 \mathrm{~d}$, where effusion takes place, to prevent its increase; $3 \mathrm{~d}$, to adopt such measures as tend to promote absorption or expectoration.

To meet the first, he recommends blood-letting generally and locally, and properly condemns the prejudices against this remedy in children. His observations on the extent to which this measure should be carried are highly judicious, and merit the particular attention of the practitioner. He seems unnecessarily anxious about the employment of purgatives, which are much less useful in this than in other disorders of children. The antimonial emetic to which he is partial is a useful remedy. But the most beneficial of all, especially when effusion is im pending or has taken place, or wherever the expediency of bloodletting is questionable, is tartar emetic, given in minute doses, so as to promote sweating and expectoration. Under this some very bad cases of pulmonary disease in children readily disappear. The paper is concluded with five cases of the disease, illustrated by dissection, and an equal number of favourable ones to demonstrate the method of treatment. 
The articles which next may be brought under notice are, the 8th, the 14th, and the 18th. The first is entitled "Cases intended to illustrate the application and utility of the Stethoscope," by Richard Townsend, A.B. M. D., to which the 18th is an appendix ; and the second consists of "Clinical observations on Phthisis Pulmonalis," by William Stokes, M. D. \&c. \&c.

These papers demonstrate the diligence with which the Dublin physicians continue to cultivate the diagnostic knowledge of pulmonary diseases; and prove, if any thing were requisite to do so, the honourable emulation with which they are mutually actuated in advancing the progress of this difficult but interesting department of medical pathology. In the Dublin Reports we had occasion to speak in favourable terms of the papers of Dr Stack, and Drs Graves and Stokes. The three articles in the present volume form valuable contributions of a similar description, and will be studied with attention by all who are interested in the knowledge of pulmonary diseases.

Of the two papers of $\mathrm{Dr}$ Townsend, the chief object is to demonstrate the utility of the stethoscope as a diagnostic agent, which is done by describing the particulars, semeiographical and necroscopical, of three cases of fatal lung disease, and the circumstances of two cases of temporary affection of the organs of respiration.

The first of these is an instance of tubercular excavation terminating in pulmonary fistula, secondary pleurisy, empyema, and pneumothorax. The existence of these lesions Dr Townsend had recognized with accuracy during life by the stethoscopic examination of the chest; and the diagnosis was confirmed by inspection after death. In the anterior surface of the left lung, about two inches from its apex, was found a round fistulous opening capable of admitting the little finger, with a defined cartilaginous margin. By the introduction of a probe, it was shown to communicate by means of a series of tubercular cavities, with a large bronchial tube. Behind, near the root of the lung, was another fistulous opening half an inch in diameter, communicating by a sinuous passage with a large tubercular excavation occupying great part of the upper lobe, and receiving also a large bronchial tube. The pleura was coated with foul, opaque, shrivelled lymph; and the air found in the cavity was ascertained by analysis to consist of

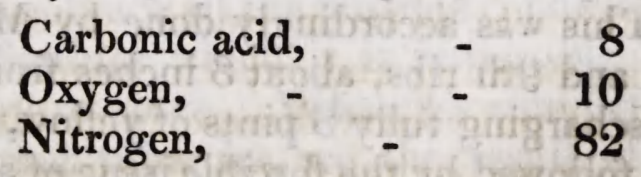

in the 100 parts, not very different from the constitution of expired air, as determined by Allen and Pepys. 
The other circumstances of this case, for instance the tuberculated state of the left lung, the enlargement of the mesenteric glands, \&c. present nothing of much moment to the accuracy of the diagnosis. The villous membrane of the stomach was quite pulpy, and those of the ileum and colon occupied with patches of ulceration.

In the second case is evinced the candour of Dr Townsend, in so far as the diagnosis was not entirely accurate. The first exploration led to the inference, that the patient had hypertrophy of both ventricles, especially the left. The second, that he had also double pneumonia. Inspection showed the heart to be of natural proportions, and though with a pervious foramen ovale, yet not such as to allow the communication of the blood of the right and left auricle. The right lung was indurated or hepatized, and occupied with miliary tubercles in different stages of growth. In the upper lobe of the left lung was a cavity as large as an orange, communicating freely with the bronchial tubes; the root of the lung was indurated by tubercles; and the rest of the pulmonic tissue was in the first stage of pneumonia.

Dr Townsend properly enough ascribes his mistake in this case chiefly to imperfect exploration of the chest. The energy with which the beats of the heart were communicated through the solidified lung tended to the inference, that the former organ was diseased. A more careful examination would in all probability have shown him, by the absence of the respiratory murmur, that the lung was really indurated and impervious to air. His failure in detecting pectoriloquy and the excavation must have arisen from no other cause than neglecting to apply the cylinder over the proper part of the chest.

The third case, which is contained in the appendix, is one of pulmonary fistula, with empyema and pneumothorax, originating, to all appearance, in pleurisy, and determined to this mode of termination by the presence of a few tubercles in the lower lobe of the right lung.

After recognizing empyema, pneumothorax, and a fistulous communication, in a young man of 20 , otherwise apparently robust, and originally of sound constitution, at the end of $\%$ days, during which his symptoms had gone on to a most aggravated degree, Dr Townsend was led, from the impending danger of asphyxia, to propose the operation of puncturing the cavity of the pleura. This was accordingly done by $\mathrm{Mr}$ Macdowell between the 8th and 9th ribs, about 3 inches from the spine, with the effect of discharging fully 3 pints of yellow, puriform, heavysmelling fluid, followed by the forcible issue of air at each expiration. By this the patient was much relieved; the metallic tinkling and amphoric humming, characteristic of the combined pre- 
sence of fluid and air, entirely ceased ; though the voice continued to reverberate as in a tubercular cavern. On the day after operation the humming had returned in a slight degree at each inspiration; but there was no tinkling till the afternoon, when, by introducing a female catheter, a pint and a half of chocolate-coloured fluid escaped in jets, which were converted into a continued stream by pressure on the abdomen. This discharge was followed as before with the escape of air, which, however, was less fetid. 'Though evident relief was the immediate consequence, the frequency of his respiration, 48 , and pulse, 140, were unfavourable indications. The breathing became exceedingly laborious, and after a restless and agitated night he expired at an early hour on the following morning.

Dissection showing the right lung collapsed to $\frac{1}{3}$ of its size, attached to the third rib through its whole length by a firm cartilaginous band, adhering also to the diaphragm, but elsewhere free. The pleura was covered by a thick layer of organized highly vascular lymph. In the middle of the lower lobe, on its posterior surface, was a large round fistulous opening, with indurated margins, capable of admitting the finger, occupying $\frac{3}{4}$ ths of an inch of the pulmonic tissue, (thickened,) lined with a smooth membrane, and communicating with a large bronchial tube. The surrounding pulmonic tissue contained from 8 to 10 gray semitransparent granular tubercles of the size of small shot. The rest of the lung was more compact and uncrepitating, yet without trace of tubercle.

Dr Townsend justly distinguishes this case of pleuro-pneumothorax from those which take place during the concluding stage of tubercular softening, by the sudden transition of the individual from health apparently robust to the agonies of an acute pulmonary disorder, threatening immediate suffocation. The primary origin of the disease involves, he justly remarks, one of the most important questions in the history of tubercles. Did the few tubercles found in the vicinity of the fistulous opening precede the pleuritic attack, and by their softening cause that inflammation? Did they precede it, and was their softening accelerated by a pleuritic attack suddenly but spontaneously, and independently induced? Or, thirdly, was the formation of these bodies altogether posterior to the pleuritic attack, and was their formation its consequence or effect? These three questions, which embrace all the possible conditions of the problem, present difficulties which it is impossible in the present state of pathology to solve. Whatever simplicity may recommend the idea that tubercles are the result of inflammation, and that, in the present case, they were the effect of the pleuritic attack, other circumstances might with equal justice warrant 
the inference, that the tubercles pre-existed, and that their softening was accelerated by the pleuritic attack. This, which is the view of the present case adopted by the ingenious author, is perhaps the correct one.

In speaking of the means of relief, Dr Townsend justly remarks, that, when the disease is known to originate in, or be connected with, tubercular disorganization, the operation of puncture of the pleura cannot be expected to be permanently beneficial. It is only in cases of empyema, resulting from genuine pleurisy, unconnected with tubercular destruction, that it can be performed with the hope of effecting a cure. It is on this account that the diagnosis of genuine pleuritic empyema, and that from the rupture of a tubercular cavity into the pleura, becomes a matter of great practical importance.

The next case in the paper of Dr Townsend, which is an illustration of the utility of the stethoscope in recognizing the state of the lungs during spasmodic asthma, may be omitted. In the last the author traces the progress of an attack of pneumonia after fever from hepatization towards resolution and convalescence.

The paper of Dr Stokes is not so remarkable for any novelties as for bringing forward some confirmations, which, however, are very valuable, of various pathological principles already advanced by Laennec, Andral, and others. It contains some cases of tubercular disease of the lungs, variously complicated with bronchial, pneumonic, or pleuritic inflammation, and with intestinal inflammation and ulceration, terminating in fatal hemorrhage.

The first case, that of a girl of 16 labouring under gastric fever, is an example of the deposition of tubercles in the thoracic and abdominal viscera generally. Not only were minute granular bodies, some like vesicles, some solid, found in the right lung, and small white tubercles in the left lung, with injection of the pulmonic tissue; but opaque white bodies of various size were found in the liver; yellowish half-softened tubercles were found in the spleen; and similar bodies, though less numerous, were found in the pancreas, the kidneys, and the womb. Though Dr Stokes admits that the large yellow tubercles in the spleen and liver were the product of some previous affection, he thinks that those in the lungs and the minute vesicular bodies in the abdominal organs were generated during the fatal illness.

It is manifest this is a point which it is impossible to determine either in the negative or in the affirmative. It is perhaps not more difficult, and, upon the whole, equally probable, to imagine, that the tubercular deposition had commenced a 
considerable time before, the minute ones perhaps subsequent to the larger; and that they had no connexion with the fatal illness, further than rendering the intimate tissue of the organs less capable of escaping without lesion, the peculiar capillary distension which attends the febrile process. Had the lungs and other organs of this girl been free from tubercles, she might have passed through the fever with impunity. As it was, the presence of the tubercles rendered the capillary injection taking place during fever more intense, and consequently more injurious.

The next two cases are examples of catarrh terminating in phthisis. It may be justly doubted, however, whether this be the legitimate title of the disease; in other words, whether the catarrhal affection could be said to terminate in tubercular softening. In the first case, which alone became the subject of necroscopic inspection, tubercular deposition was found in both lungs, an excavation in the upper portion of each, and many of those in the right lung softened; the intermediate pulmonic tissue was red and soft; while that of the left was solid and reddish-gray; and the bronchial membrane was red and firm, without ulceration. Now the catarrhal phenomena with which this case is said to have commenced, are precisely the effects which result from the incipient softening of tubercles, whatever be the remote cause which induces that softening. A person labouring under tubercular disease of one or both lungs is very susceptible of what is called cold; in other words, is very frequently harassed with cough and expectoration, which is at this period mucous only. He naturally ascribes it to exposure to cold; and in truth, exposure to a degree of cold, which has no effect on an individual with sound lungs, makes a most serious impression on one with tubercular deposition. In process of time, either with or without such exposure, for the natural tendency of tubercles is, at a certain period of their developement, to soften, this process takes place, and of itself induces, totally independent of cold, inflammation, not only of the pulmonic tissue, but of the adjoining bronchial membrane, and its usual consequence cough and mucous expectoration. 'That this is the natural, and, as it may be termed, the necessary course of tubercular disease in the lungs, may be demonstrated to a certainty by many facts. In several cases of tubercular softening and excavation which we have had occasion to examine with care, we never failed to observe more or less redness and thickening of the bronchial membrane, not unfrequently complete erosion, with an unusual quantity of frothy serous mucus, the product no doubt of the inflamed membrane. The stethoscopic indications even announce this as the ordinary course of phenomena. The mucous rattle of catarrh both pre- 
cedes and accompanies the process of softening; and even after pectoriloquy is distinct and the expectoration becomes tubercular, the mucous gurgling in the chest continues, and a large proportion of the expectoration is mucous. In short, it may be stated as a uniform principle of pulmonary pathology, that no tubercle undergoes the process of softening without causing inflammation, at least of the contiguous bronchial membrane, and very frequently of the pulmonic tissue. This is the genuine origin of the catarrh which is said to terminate in phthisis, a confused and inaccurate mode of expression arising from inattention to the pathological process in which the catarrhal affection depends. These observations are not adduced as new or unknown; for we believe that the ingenious author of the paper before us must be perfectly aware of the facts on which they are founded. But as some misconception appears still to prevail in the relation between phthisical or tubercular catarrh and the disorganizing process, which is not its termination but its cause, this we conceive is not an unseasonable opportunity for endeavouring to place the matter in its just light.

The fourth case in the present paper is most remarkable for illustrating the morbid state of the intestinal tube in the phthisical. The pulmonary phenomena and necroscopic appearances are similar to those of many other cases. The individual had voided at stool about $2 \frac{1}{2}$ quarts of fluid blood, and soon after expired. Besides softening of the stomach and enlargement of the muciparous glands of the duodenum, the jejunum presented many, and the ileum fewer circular patches of ulceration with raised edges, evidently the mucous membrane. The iliocœeal portion presented many old cicatrices. The ascending portion of the colon was studded with enlarged glands, and presented numerous ulcerated patches, in the interval of which the mucous membrane was thickened and elevated. Though the transverse portion appeared healthy, at the beginning of the sigmoid flexure " was a large patch of recent ulceration extending completely round the intestine. On each side of this patch the mucous membrane had the appearance of ecchymosis observed in the stemach in cases of hæmatemesis." It is very probable that this was the source of the fatal hemorrhage.

Dr Stokes judiciously directs the attention of the practitioner to the fact, that the administration of opiates and astringents, so often pursued in this state of the intestines, which may augment the fever, and accelerate the fatal result, ought to give place to a mode of treatment which proposes to abate inflammation of the mucous membrane and glands, and thereby obviate ulceration. On this head we may be allowed to say from experience, that astringents are invariably injurious in the diarrhoea of the 
phthisical, sometimes to the degree of inducing acute intestinal inflammation; and that the remedial measures which are most beneficial are small doses of castor oil, alternating with the use of the starch injection.

The fifth case may be regarded as affording some confirmation of the opinion advanced by Broussais, and recently defended with much ingenuity by $\mathrm{Dr}$ Alison of this place,-that tubercle is the result of inflammation. In a man of 45 , who had taken mercury for a syphilitic affection, symptoms of pneumonia appeared after exposure to cold. These symptoms were aggravated by subsequent injury of the chest, by which three ribs were broken; and from this he had scarcely recovered, when a heavy weight falling on his shoulders, and bending him forcibly torward, was again followed by a recurrence of the pectoral symptoms, for which he was admitted into the Meath Hospital on the 26th June 182\%. Two days after this he was found to have a spot of cavernous respiration, with strong resonance in the upper part of the right breast, to which was added the following day imperfect metallic tinkling. He went home, and expired ten days after. The whole upper lobe of the right lung was converted into a large abscess, the walls of which consisted of hardened pulmonic tissue, and were void of the usual cartilaginous lining of the tubercular excavation. Masses of tubercular matter, however, of various sizes, some softening in the centre, were scattered through the lung. The intermediate tissue was hard and reddish gray. In the upper part of the left lung were a few tubercles, and the bronchial membrane was tinged of a deep red colour.

It is remarked by the author, that the production of tubercles in this case was probably preceded by pneumonia, and induced by continued irritation. To this opinion the chief objection consists in the tubercles, which, though few, were found in the left lung, which had not suffered from mechanical injury, and in which, if inflammation did cause the tubercular deposition, it must have arisen from the exposure to cold during the mercurial influence. To this view, indeed, Dr Stokes afterwards adverts, and takes occasion to reprobate the practice of repeated mercurial courses in persons in whom phthisis is likely to take place.

The last case is an example of fistulous opening into the pleura, with empyema and pneumothorax. In every respect, both semeiographical and necroscopic, it bears so close a resembling to the first of Dr 'Townsend, that it is unnecessary to enter into any detailed account. The tubercular destruction affected both lungs; but the fistulous opening was confined to the left.

Under the same head we turn to the case, or rather cases, 
for there are two, of open Foramen ovale, given by Dr John Crampton.

The first occurred in a young man of 18 , who was admitted into the Hardwicke Hospital as a fever patient on the 6th February 182\%. From the febrile symptoms he had scarcely recovered, when he was attacked with those of acute rheumatism; and to these again succeeded symptoms denoting disease of the lungs and heart. The stethoscope indicated acute bronchial inflammation with hypertropy and irregular action of the heart. The cardiac symptoms became successively worse; and he expired on the 10th of March, a little more than a month after admission.

With traces of inflammation of the pericardium, the ventricles were found enlarged; the semilunar aortic valves edged with recent fleshy excrescences; the aortic orifice contracted; that of the pulmonary artery enlarged; the auricles dilated; and in the auricular septum an oval depression presenting an oblique or valvular communication as large as the calibre of a goose quill.

In the second case, a man aged 34, in the Whitworth hospital, died with symptoms of phthisis. Besides hepatized and tuberculated lungs, and a large excavation in the left lung, the foramen ovale was found open; but the valvular arrangement of the auricular membrane was such, that in ordinary circumstances no communication between the blood of the two auricles could take place.

A degree of openness of the foramen ovale, it may be observed, is not uncommon in the bodies of those who die of tubercular disease of the lungs.

The last paper which we shall at present notice is the 66 Sketch of a Medical Report on the Epidemic Dysentery which prevailed in Dublin in the year 1825, by Dr John O'Brien." It was chiefly in the autumn and early part of the winter of this year that dysentery was epidemic in Dublin. Whether the extreme heat and drought of the summer and autumn of this year could have any influence in the production and diffusion of this disease, certain it is, dysentery, commencing first among the better classes, proceeded with rapid and extended strides to affect the labouring classes. It was about the 15th of August that the registry of Cork Street Hospital, by the increased number of admissions, afforded unequivocal proof of the epidemic diffusion of intestinal disease. After this period it increased rapidly till the beginning of November, when it seemed to have attained its maximum. The account of $\mathrm{Dr}$ O'Brien may be given in his own words.

"The duration of the disease when it terminated favourably varied from 2 to 6 weeks; and of the fatal cases 4 died in the 
acute stage, that is within the first three weeks of the disease, and five lingered on to the chronic state, under which they ultimately sunk. The ages of the majority varied from 20 to 40 ; none were under the age of 20 ; and 2 only (fatal cases) exceeded the age of 40. It was not a little remarkable that persons of middle age, and in the vigour of life, seemed to have been the principal sufferers in the present epidemic, and that the young and aged escaped in a greater proportion than usual. In the males, it may be observed, the mortality was less than in the females."

The disease was generally attended with febrile symptoms. But, as in all similar cases, it sometimes happened, that the febrile symptoms preceded the dysenteric so far that the disease was taken for fever; in other cases the dysenteric disorder was actually a consequence or sequel of the fever. In one or two instances only was there any ground for the suspicion of contagion.

The pain, which was in general seated in the prominent part of the hypogastric region, a little below the navel, was more frequently fixed than intermitting. The discharges were dark brown, sometimes approaching to tar, and in one or two cases like coffee-grounds.

The general plan of treatment appears to have been bloodletting to the extent of 12 or 14 ounces, followed by immersion in the warm bath, frictions of the person, but especially of the belly, with camphorated oil, and swathing by a flannel roller; and, lastly, ten grains of calomel with two grains of opium, and occasionally antimonial powder. This course on the first evening of treatment was followed next morning by a purgative draught consisting of castor oil, tincture of rhubarb, and tincture of opium, or of infusion of senna and salts with tincture of rhubarb and tincture of opium. If the symptoms did not give way, the blood-letting and the warm bath were repeated, and the calomel and opium, with purgatives, were continued till the gums became tender, after which the exhibition of opium alone generally effected a cure. It is amusing to find the author gravely giving his testimony in favour of the non-irritating qualities of castor oil, which it appears some practitioners in that country regard as an acrid substance. To such an opinion nothing but prejudice and ignorance could give rise. No man who ever administered castor oil in half a dozen or half a score of cases could ever continue to entertain it.

The most valuable part of the paper is the abstract of the dissections at Sir P. Dun's Hospital. The descriptions are so similar to those of all other cases of dysentery that we need not repeat them in detail. It is sufficient to say, that in 8 of 12 cases the villous membrane of the small intestine, prin- 
cipally the ileum, was inflamed or ulcerated in patches; that in the whole twelve the colon was vascular, inflamed, ulcerated, eroded, or otherwise diseased; that in one it was gangrenous, and in two much contracted; that the rectum was much inflamed and abraded; and that the liver was diseased in six and the spleen in three. In all the cases also the peritonæum contained more or less serous or sero-albuminous fluid.

The above papers form the principal ones on subjects in medical pathology. We should now proceed with the examination of the others, which are highly interesting and not less deserving regard. This, however, we must defer for the present, in favour of other matters which claim consideration. We, therefore, close the volume, with the hope of soon recalling the attention of our readers to subjects on which we cannot doubt they feel as much interest in receiving information as the industrious members of the Dublin Institution have in communicating it.

Art. III.-Surgical Observations on the Treatment of Chronic Inflammation in various Structures, particularly as exemplified in the Diseases of the Joints. Вy Jонn Scort, Surgeon to the London Ophthalmic Infirmary, and AssistantSurgeon to the London Hospital. London, 1828. 8vo. pp. 291.

THE treatment of chronic inflammation, and its consequences in the textures forming the different articulations, has long been a task of such extreme practical difficulty, as in a large proportion of cases to make it be abandoned for the more certain and less tedious alternative of removal by the knife. When a joint affected with incipient disease is submitted to the surgeon, the first proceeding is to pursue the usual routine measures, the chief object of which is to check inflammation and to obviate its products. The local detraction of blood, repeated according to its apparent effects, the application of blisters, tartar emetic, or sulphuric acid ointment, caustics, or moxa to produce ulceration, setons and other counter-irritants, not omitting dry friction and the douche, are the means usually tried with the view of subduing the inflammatory action in the capsular cellular membrane, in the synovial membrane or articular glands, or in the cartilaginous extremities of the component bones. When these resources of surgery are exhausted unavailingly, the joint, if removeable by amputation, is consigned to the knife; or if beyond the reach of this alternative, as in the vertebræ and the 